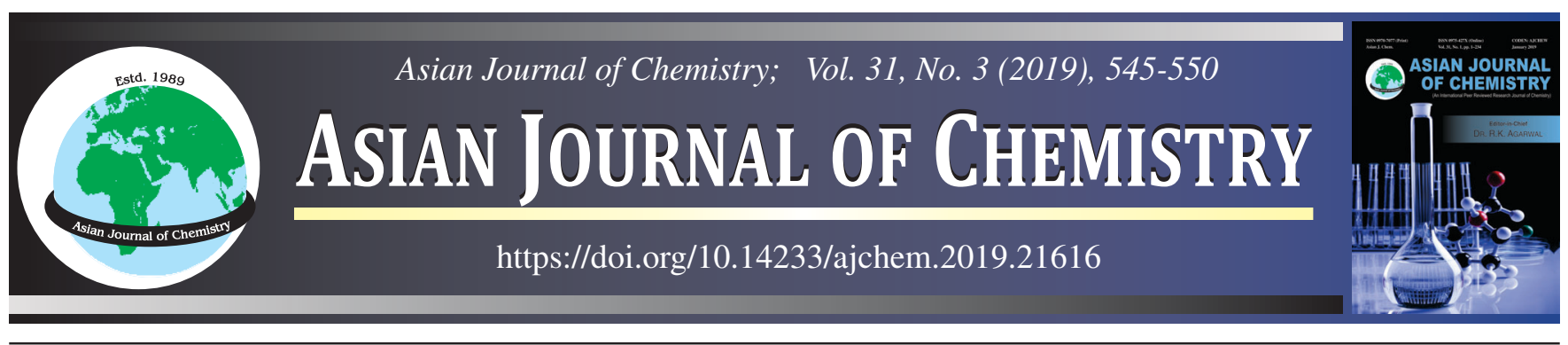

\title{
A Precised Surface Modification of Hydroxyapatite with Poly(methylmethacrylate) for Tissue Engineering \& Regenerative Medicine
}

\author{
Trinh Duy Nguyen ${ }^{1, \bullet}$, Phu Thuong Nhan NGuyen ${ }^{1}$, Thien Hien Tran ${ }^{1}$, \\ Md. Rafiqul Islam ${ }^{2}$, Kwon Taek Lim ${ }^{2}$ and Long Giang BaCh ${ }^{1,3,4, *, \bullet}$
}

${ }^{1}$ NTT Hi-Tech Institute, Nguyen Tat Thanh University, Ho Chi Minh City 700000, Vietnam

${ }^{2}$ Department of Display Engineering, Pukyong National University, Busan 608-737, Republic of Korea

${ }^{3}$ Graduate University of Science and Technology, Vietnam Academy of Science and Technology, Ha Noi City 100000, Vietnam

${ }^{4}$ Center of Excellence for Functional Polymers and NanoEngineering, Nguyen Tat Thanh University, Ho Chi Minh City 700000 , Vietnam

*Corresponding author: Fax: +84 2839404759; Tel: +84 969294297; E-mail: blgiangntt@gmail.com; blgiang@ntt.edu.vn

The poly(methylmethacrylate) (PMMA) grafted biocompatible hydroxyapatite nanocrystals (HAPs) hybrid nanocomposites (PMMA-gHAPs) were synthesized by employing surface thiol-lactam initiated radical polymerization (TLIRP) through grafting from strategy. At first, the surface of HAPs was functionalized by 3-mercaptopropyl-trimethoxysilane in one-step process to prepare thiol immobilized HAPs (HAPs-SH). Subsequently, a controlled radical polymerization of MMA by using two component initiating system comprising of HAPs-SH and butyrolactam (BL) successfully afforded PMMA- $g$-HAPs nanocomposites. The resulting structure and morphological feature of nanocomposites was systematically characterized by FT-IR and XRD analyses. GPC studies of cleaved polymers from nanocomposites of different time revealed that the grafting polymerization from the surface of HAP was well controlled in nature. Moreover, the thermal property of the PMMA was found to be improved by incorporation of inorganic HAP nanoparticles in the polymer matrix as revealed by TGA and DSC studies. The colloidal stability of the synthesized nanocomposites was observed to be exceptionally good in organic solvents as suggested by the time dependent monitoring using UV-visible spectroscopy and captured digital photographs. The synthesized nanocomposites show a great promise for the safe application in tissue engineering and regenerative medicine.

Keywords: Hydroxyapatite, Poly(methyl methacrylate), Surface initiated radical polymerization, Hybrid nanocomposites, TLIRP.

ᄂ _ - - - - - - - - - - - - - - - - - - - - - - - - - - - - - - - - - -

\section{INTRODUCTION}

Preparation of novel materials with fascinating properties and improved performance is a continuously expanding research field, which covers subjects ranging from chemistry, physics, biology and material science. Polymer-inorganic nanomaterials are recognized as a hot spot of current research because of their extraordinary hybrid properties. They integrate the characteristic features of inorganic nanostructures with expected properties of polymers, for example, reduced toxicity, improved dispersibility and chemical stability and preventing agglomeration of inorganic particles. Combination of active organic and inorganic components at the nanosized level is a great challenging task of current materials science. These functional hybrids are considered as advanced materials with promising applications in many fields such as medicine, drug delivery, electronics, energy storage and conversion, mechanics, catalysis etc. [1-5]. Recently, several strategies have been developed for the synthesis of biomaterials, which cover a rage of applications in biomedicine. Presently, hydroxyapatite nanocrystals, $\mathrm{Ca}_{10}\left(\mathrm{PO}_{4}\right)_{6}(\mathrm{OH})_{2}$, have attracted a significant attention due of the resemblance of chemical properties with the natural $\mathrm{Ca}_{3}\left(\mathrm{PO}_{4}\right)_{2}$ present in tissue as bones and teeth. The hydroxyapatite nanocrystals (HAPs) plays an important in upholding the mechanical features of bones and provide a coherent surroundings for biocompatibility, bioactivity, solubility, castability, sinterability, fracture toughness and absorption ability. Many studies show that the link between structural and applied in biology as bone filler, orthopedic emned and polymeric filler. Considering the promising applications of HAPs, many efforts have been undertaken on the modification of HAPs [6-14].

This is an open access journal, and articles are distributed under the terms of the Creative Commons Attribution-NonCommercial-ShareAlike 4.0 (CC BY-NC-SA 4.0) International License which allows readers to freely read, download, copy, distribute, print, search, or link to the full texts of its articles and to use them for any other lawful non-commercial purpose as long as the original source is duly acknowledged. 
In many different alterations, the attachment of non-inorganic polymers on the surface of HAPs has become the center, which has synergistic effects on each component with a variety of coupling agents and polymers. A number of controlled/living radical polymerization techniques, such as atom transfer radical polymerization (ATRP), nitroxide-mediated polymerization (NMP) and reversible addition-fragmentation chain transfer polymerization (RAFT) have some advantages over control of the architecture, molecular weight distribution in comparison with the conventional radical polymerization [15-20]. For all that, most of the techniques require many reaction steps to introduce functional groups to the inorganic surface for polymerization with complex structures and/or it is confusing to remove the metallic catalyst all through from the products.

On the other hand, because of a great chain transmission constant so surface guaranteed -SH species are used as chain transfer agents in unattached radical polymerization. The use of 3-mercaptopropyl-trimethoxysilane (MPTMS) as a silane binder performs a surface chain-transfer response to produce extremely thin polymer films, as in reported of Zhou et al. [21]. 3-Mercaptopropyl-trimethoxysilane is used as a chain-switching agent in the manufacture of polymer attached inorganic particles. The creation of a compound of comonomers of chemical substances as methyl methacrylate/N-cyclohexylmaleimide is accomplished through polymerization, can only begin with the -SH group. During polymerization, the presence of large amounts of styrene, methyl methacrylate with $n$-dodecylmercaptan and $\varepsilon$-caprolactam or 2-pyrrolidinone taken to in the beginning of the monomer synthesis [22-26]. Recently, we found a facile strategy for fabricating inorganic particles based on thiol group with the influence of butyrolactam (BL) [27-30].

Taking the above discussion into accounts, it would be an attractive strategy employing a one-step direct anchoring of initiator moiety, preferably thiol groups, on HAPs surface that eventually resulted in the hybrid HAPs nanocomposites with well-defined high density poly(methyl methacrylate) (PMMA) polymer. PMMA is a material commonly used as bone cement for fixing total hip prostheses to offer suitable mechanical properties to the material. Initially, treatment of MPTMS with HAPs would result HAPs-SH upon the reaction between trimethoxysilane groups of MPTMS with the -OH species on the surface of HAPs via ligand-exchange reaction. Subsequent grafting polymerization of MMA from HAPs-SH surface was accomplished in the presence of BL to afford PMMA- $g$-HAPs nanocomposites. Herein, this technique is recognized as thiollactam-initiated radical polymerization (TLIRP). Based on previous studies, we found that it is the first study to synthesize the chemically bonded MMA with HAPs using such a facile living radical polymerization technique employing grafting from strategy.

\section{EXPERIMENTAL}

Using a neutral alumina column to evict the inhibitor in methylmethacrylate (MMA) before to use. Hydroxyapatite nanocrystals (HAPs), (3-mercaptopropyl) trimethoxysilane (MPTMS), butyrolactam (BL) and all solvents were used as received, which were purchased from Aldrich.
Synthesis of PMMA-g-HAPs nanocomposites via surfaceinitiated TLIRP: The surface of HAPs was coated with MPTMS by a silanization reaction to obtain thiol functionalized HAPs according to the reported procedure [31]. A typical procedure for synthesis of PMMA- $g$-HAPs nanocomposites by TLIRP is described as: $0.0002 \mathrm{~kg}$ of HAPs-SH, $0.001 \mathrm{~kg}$ of MMA, $0.0004 \mathrm{~kg}$ of BL, $0.002 \mathrm{~L}$ of THF and using a Teflon-covered propulsion rod were put in a $0.025 \mathrm{~L}$ circle bottle decorated with a restore condenser. The bottle was washed with nitrogen, heated to $90^{\circ} \mathrm{C}$ and continue stirring. At the end time, the viscosity rised substantial. End of the desired time, the temperature of bottle was reduced to $35^{\circ} \mathrm{C}$ and using excess hexane to precipitate the reaction blend. The product was removed residue and using a vacuum oven to dehydrate. Toluene is used to reduce the concentration of the product then got the polymer attached HAPs free from the unconnected polymer and then centrifuged to gather the PMMA- $g$-HAP nanocomposites.

Cleavage of the grafted PMMA from PMMA- $g$-HAPs nanocomposites: To investigate the number average molecular weight $\left(\mathrm{M}_{\mathrm{n}}\right)$ and polydispersity index $\left(\mathrm{PDI}=\mathrm{M}_{\mathrm{w}} / \mathrm{M}_{\mathrm{n}}\right)$ of the grafted PMMA chains as a function of reaction time, PMMA chains were cleaved from the surface of HAPs. In a typical method, $0.1 \mathrm{~g}$ of the PMMA- $g$-HAPs nanocomposites was treated with acetic acid $(0.5 \mathrm{~mL})$ in THF $(5 \mathrm{~mL})$ for 1 day. After separation in the organic film, PMMA is precipitated by hexane [32]. After removing HAPs by centrifugation (10.000 rpm), the supernatant was dried and dissolved in THF for GPC analysis.

The changes (with respect to chemical bonding) in the surface of HAPs, functionalized HAPs and PMMA- $g$-HAPs nanocomposites were investigated by Fourier transformed infrared spectrophotometry (a BOMEM Hartman \& Braun FTIR Spectrometer) in the frequency range of $4000-400 \mathrm{~cm}^{-1}$. The differential scanning calorimetry (DSC) measurements were operated using a Perkin Elmer calorimeter DSC6200. The crystallographic state of functionalized HAPs was determined by a Philips X'pert-MPD system diffractometer with $\mathrm{CuK}_{\alpha}$ radiation. Gel permeation chromatography (GPC) was observed using an Agilent 1200 Series equipped with PLgel $5 \mu \mathrm{m}$ MIXED-C columns, with tetrahydrofuran (THF) as the solvent at $30^{\circ} \mathrm{C}$ and calibration was carried out using PMMA standard. To examine the dispersion stability of the PMMA$g$-HAPs, the nanocomposites were dispersed in chloroform by a sonicator for $10 \mathrm{~min}$. The dispersibility and colloidal stability were evaluated using a Perkin-Elmer Lambda 40 ultravioletvisible spectrophotometer.

\section{RESULTS AND DISCUSSION}

The chemical immobilization of MPTMS on the surface of HAPs and the grafting of PMMA on HAPs surfaces were studied by FT-IR spectroscopy. The FT-IR spectra of the HAPs and HAP-SH are shown in Fig. 1. In the case of the HAPs (Fig. 1A), the strong absorption bands at 1032 and $958 \mathrm{~cm}^{-1}$ are assigned to the stretching of phosphate $\left(\mathrm{PO}_{4}{ }^{3-}\right)$ species and strips at 556 and $606 \mathrm{~cm}^{-1}$ are accountable for distortion vibrations of $\mathrm{PO}_{4}{ }^{3-}$ species. The absorption strip at $3566 \mathrm{~cm}^{-1}$ is attributed to the unbound - $\mathrm{OH}$ species stretching vibration in crystal lattice of the HAPs phase. The less intense absorption 


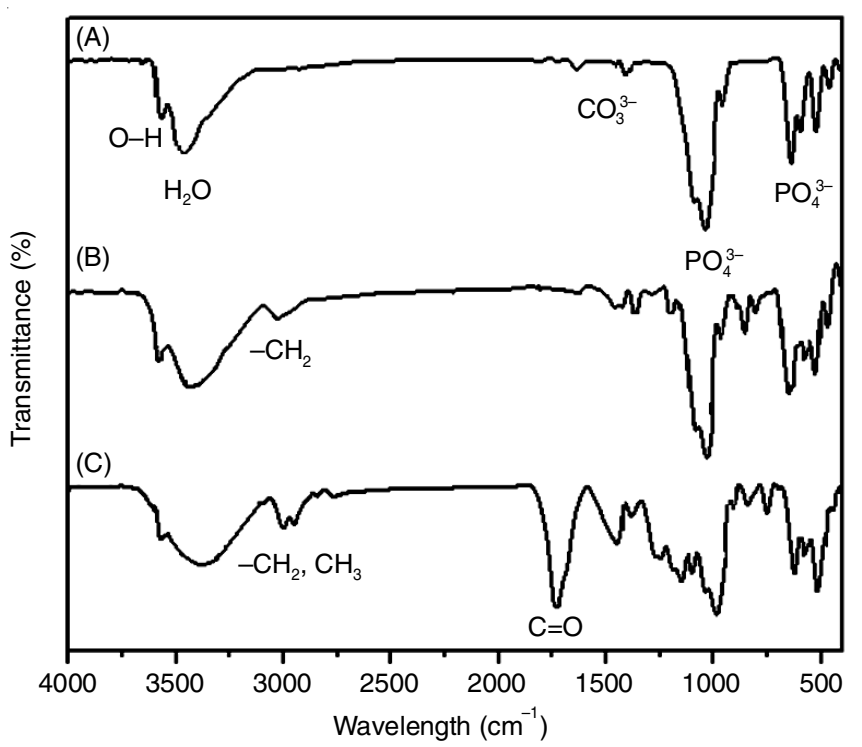

Fig. 1. FT-IR spectra of (A) HAP nanocrystals, (B) HAP-SH and (C) PMMA- $g$-HAP nanocomposites

band at 1634-1601 $\mathrm{cm}^{-1}$ (bending vibrations) and the large strip at $3407 \mathrm{~cm}^{-1}$ (stretching vibrations) are assigned of $\mathrm{H}_{2} \mathrm{O}$ adsorbed on the HAPs. Weak bands of carbonate $\left(\mathrm{CO}_{3}{ }^{3-}\right)$ at $1448-1417 \mathrm{~cm}^{-1}$ also exhibit in the spectrum indicating the carbonate-hydroxyapatite was afforded [33]. In the FT-IR spectrum of HAPs-SH as shown in Fig. 1B, a new weak band in $3000-2900 \mathrm{~cm}^{-1}$ region is appeared because of the aliphatic $\mathrm{C}$-H stretching of the $\mathrm{CH}_{3}$ - and $\mathrm{CH}_{2}$ - groups of MPTMS residues. FT-IR spectrum provides qualitative information on chemical bonding in the PMMA-g-HAP nanocomposites as shown in Fig. 1C. The absorption spectrum of PMMA- $g$-HAP nanocomposites is somewhat similar with that of HAP nanocrystals, such as a broad adsorption strip at $3396 \mathrm{~cm}^{-1}$ is accountable for bending and stretching of $\mathrm{H}_{2} \mathrm{O}$ adsorbed in the HAP, the strong absorption bands at 1028 and $962 \mathrm{~cm}^{-1}, 562$ and 603 $\mathrm{cm}^{-1}$ are ascribed to the stretching of $\mathrm{PO}_{4}{ }^{3-}$ species and the deformation of $\mathrm{PO}_{4}{ }^{3-}$ species, respectively. In addition, the characteristic bands in the variety of 3000 to $2800 \mathrm{~cm}^{-1}$ and potent band at $1735 \mathrm{~cm}^{-1}$ might ascribe for $\mathrm{C}-\mathrm{H}$ stretching of the $\mathrm{CH}_{3}$ and $\mathrm{CH}_{2}$ species and the $\mathrm{C}=\mathrm{O}$ species stretching of PMMA on the surface of HAP particles, respectively. In the FT-IR spectrum, therefore, a PMMA chain is clearly recognizable. These results suggest that the PMMA was successfully grafted on the surface of HAP particles [34].

Controlled radical polymerization from HAP-SH nanocrystals was tested for various reaction time varying from 1 to $9 \mathrm{~h}$. Without using butyrolactam, TLIRP approach was attempted under the similar condition but was not successful. However, when butyrolactam was added in the system, the situation was improved dramatically and hybrid products with high molecular weight were obtained in high yield. The polymerization presumably proceeded via fundamental adding with unsteady HAP-S ${ }^{\bullet}$. In contrast, the labile $\mathrm{BL}-\mathrm{H}^{\bullet}$ radical might terminate the polymerization method and led to the driving of the polymerization. At the initial stages of the TLRIP, the solution was slightly turbid because of the low dissolution of HAP-SH in the response medium. The solution gradually became transparent upon polymerization proceeded, this phenomena might be because of the progressive immobilization of PMMA chains on the HAP surface. The monomer conversion was reached at about $66.1 \%$ after polymerization for $9 \mathrm{~h}$. In order to characterize the grafted PMMA and whether the TLIRP polymerization was controlled, the PMMA- $g$-HAP nanocomposites were dissolved in toluene and subsequently the PMMA grafts were detached using acetic acid. The PMMA brushes were isolated from the solution and subjected for analysis by GPC. Fig. 2A demonstrates the plots of the number average molecular weight $\left(\mathrm{M}_{\mathrm{n}}\right)$ and molecular weight distributions $\left(\mathrm{PDI}=\mathrm{M}_{\mathrm{w}} / \mathrm{M}_{\mathrm{n}}\right)$ of the grafted PMMA with polymerization time against the overall monomer conversions. In GPC trace, it is observed that the $\mathrm{M}_{\mathrm{n}}$ of PMMA increased with increasing monomer conversion as well as polymerization time suggesting the TLIRP as controlled technique. The GPC traces indicate that the PDIs are unimodal and narrow (Fig. 2B). The GPC analysis of the cleaved PMMA brushes (polymerization time $9 \mathrm{~h}$ ) reveals that the $\mathrm{M}_{\mathrm{n}}$ and PDI are 90,800 and 1.77, respectively.
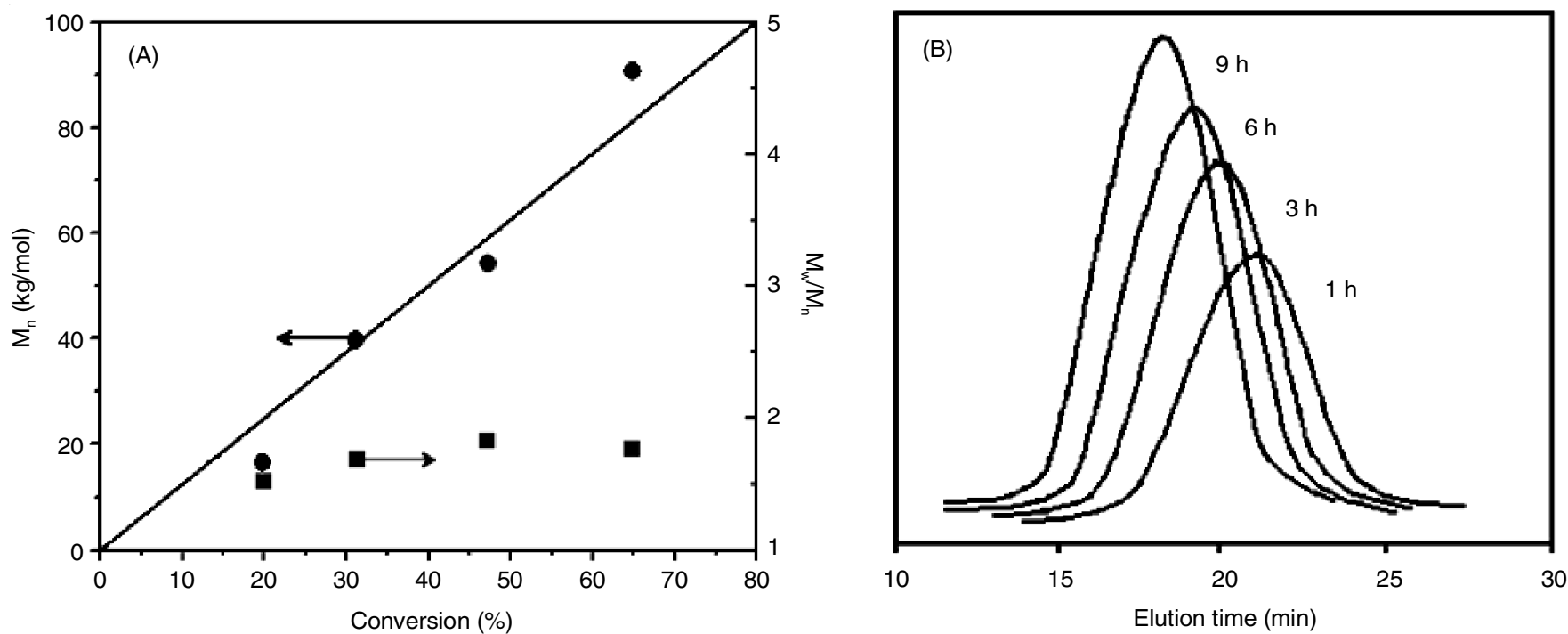

Fig. 2. (A) $M_{n}, M_{w} / M_{n}$ and (B) $M_{w} / M_{n}$ curves for polymerization of MMA from HAP-SH surface by TLIRP technique at different polymerization time. The PMMA was cleaved from PMMA- $g$-HAP nanocomposites using acetic acid 
Thermogravimetric analysis was employed to determine the amount of MPTMS grafted onto HAPs (Fig. 3). The sample of HAP nanoparticles shows a weight decline of about $1.9 \%$ in the region from 50 to $800{ }^{\circ} \mathrm{C}$ because of the decrease of aqua molecules adsorbed on the surface and the liberation of the aqua structural caused from the bonded -OH species (Fig. 3A). The weight decline of HAPs-SH is estimated to be about $6.2 \%$ in the range of $50-800{ }^{\circ} \mathrm{C}$ (Fig 3B). The amount of the grafted MPTMS is determined to be about $4.3 \%$. TGA was used to quantify the grafted PMMA on the HAPs at different polymerization time. The as prepared PMMA- $g$-HAP nanocomposites show that the thermal decomposition started at $285^{\circ} \mathrm{C}$ and constant weight loss was achieved at $420^{\circ} \mathrm{C}$, which may be due to the initial decomposition of grafted PMMA. In accordance with reaction time, the PMMA graft density was found to be increased linearly (Fig. 3C-F). In the TGA traces, it is seen that the PMMA weight content was changed from 27.1 to $75.1 \%$ upon the polymerization time of 1 to $9 \mathrm{~h}$. The TGA results suggest a moderate degree of functionalization of HAP particles by PMMA using grafting from strategy. The synthesized PMMA- $g$-HAP nanocomposites have showed significantly higher thermal stability than the parent polymer. Therefore, it can be recommended that TLIRP is a good attach polymerization approach for monomers with $\mathrm{CH}_{2}=\mathrm{CH}$-group on inorganic materials.

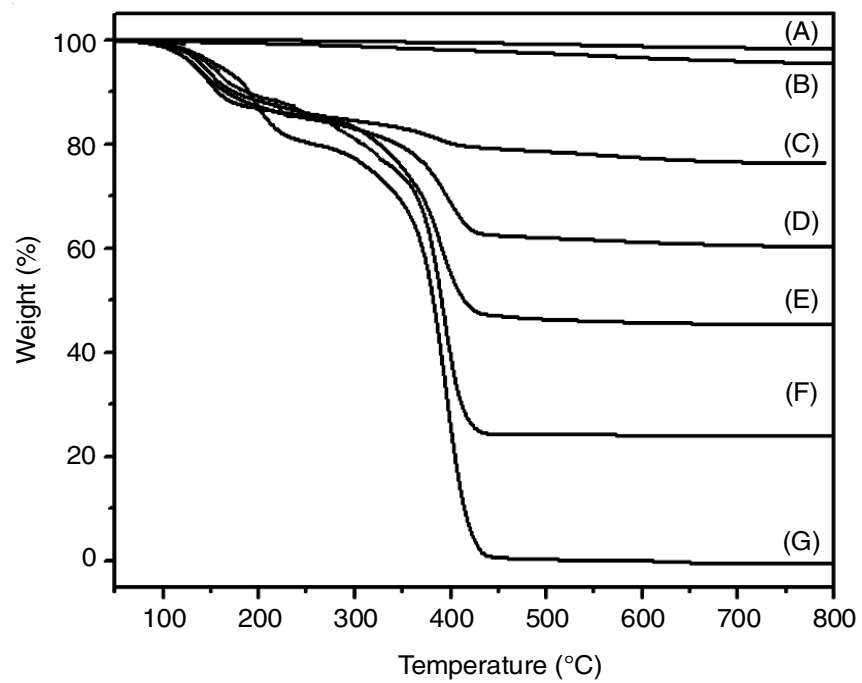

Fig. 3. Thermograms of (A) HAP nanocrystal, (B) HAP-SH, (C, D, E, F) PMMA- $g$-HAP after polymerization time of $1 \mathrm{~h}, 3 \mathrm{~h}, 6 \mathrm{~h}$ and $9 \mathrm{~h}$, respectively and (G) grafted PMMA cleaved from PMMA- $g$-HAP nanocomposites

An improved glass transition temperature $\left(\mathrm{T}_{\mathrm{g}}\right)$ of these organic-inorganic hybrid materials was observed for all the PMMA- $g$-HAP nanocomposites compared to the pure PMMA. Fig. 4 shows the $T_{g}$ values of the pure PMMA and the grafted PMMA chains onto HAPs as investigated by DSC. The pure PMMA exhibited a heat flow change at about $109.1{ }^{\circ} \mathrm{C}$, corresponding to the $T_{g}$ of PMMA. On the other hand, the $T_{g}$ of PMMA- $g$-HAP at different polymerization time was found to be $1 \mathrm{~h}\left(115.4{ }^{\circ} \mathrm{C}\right), 3 \mathrm{~h}\left(117.2^{\circ} \mathrm{C}\right), 6 \mathrm{~h}\left(121.7^{\circ} \mathrm{C}\right)$ and $9 \mathrm{~h}$ $\left(124.0^{\circ} \mathrm{C}\right)$. It was observed that $\mathrm{T}_{\mathrm{g}}$ of PMMA- $g$-HAP improved in accordance with the increased PMMA graft density. The cause behind the growth of $T_{g}$ values can be explained in such a way that the confinement of one end of the PMMA chains

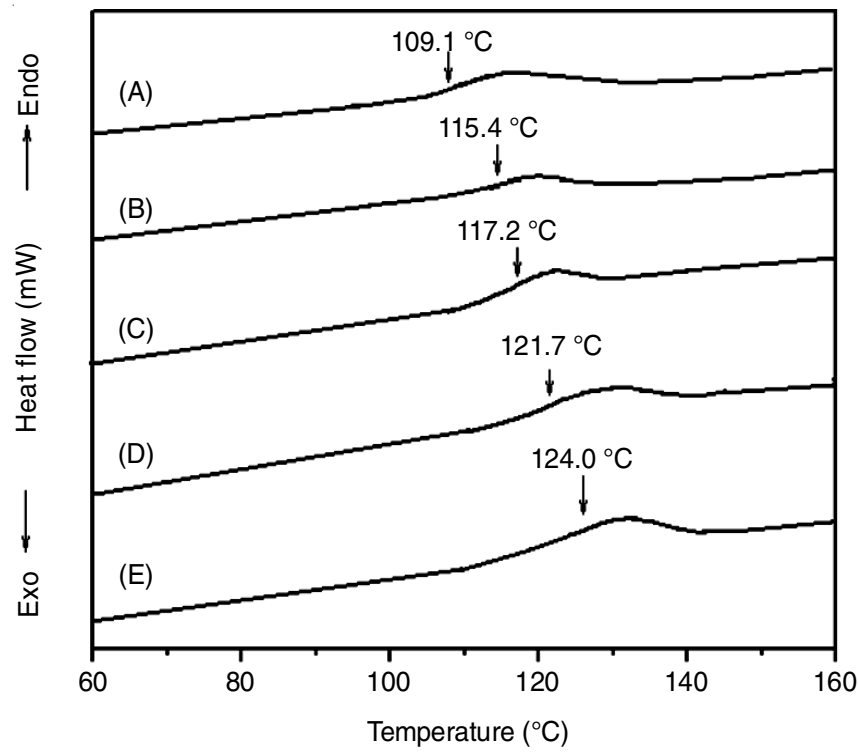

Fig. 4. DSC curves of (A) PMMA, PMMA-g-HAP after polymerization time of (B) $1 \mathrm{~h},(\mathrm{C}) 3 \mathrm{~h}$, (D) $6 \mathrm{~h}$ and (E) $9 \mathrm{~h}$, respectively

on the HAP would restrict the movement and vibration of the whole chain as developed strong interaction along with inherent high modulus of HAP resulting in increased $\mathrm{T}_{\mathrm{g}}$ [35].

The XRD pattern was used to investigate the crystallinity of the HAPs, HAPs-SH and PMMA- $g$-HAPs as shown in Fig. 5. The diffracted curves of HAPs exhibit (Fig. 5A) several sharp peaks at $2 \theta$ regions of $26^{\circ}, 29^{\circ}, 32-34^{\circ}, 40^{\circ}$ and $46-54^{\circ}$, which are full agreement with the crystalline nature of HAPs. As shown in Fig. 5B-F, the characteristic diffraction peaks of the (002), (102), (211), (300), (202), (310), (222), (213) and (411) are crystal planes for HAP-SH and PMMA- $g$-HAP nanocomposites which are almost identical with the XRD curves of HAPs. This result suggests that the grafting reaction did not cause any change in crystallinity or secondary phases, this feature might happen due to the chemical reaction and the subsistence of crystalline properties of HAPs [36].

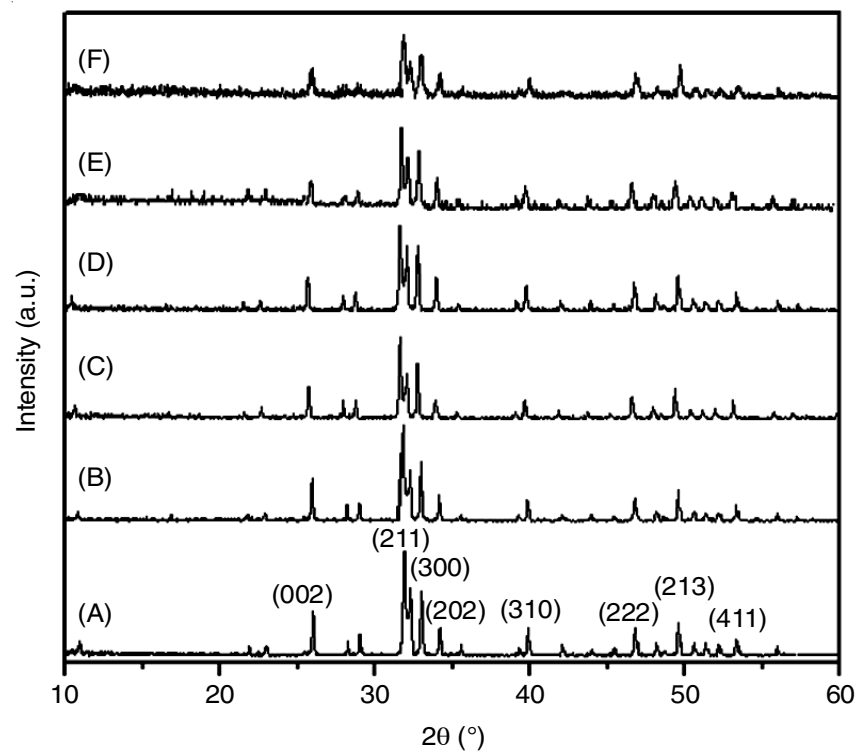

Fig. 5. X-ray diffraction curves of (A) HAP nanocrystal, (B) HAP-SH and (C, D, E, F) PMMA- $g$-HAP after polymerization time of $1 \mathrm{~h}, 3 \mathrm{~h}, 6$ $\mathrm{h}$ and $9 \mathrm{~h}$, respectively 

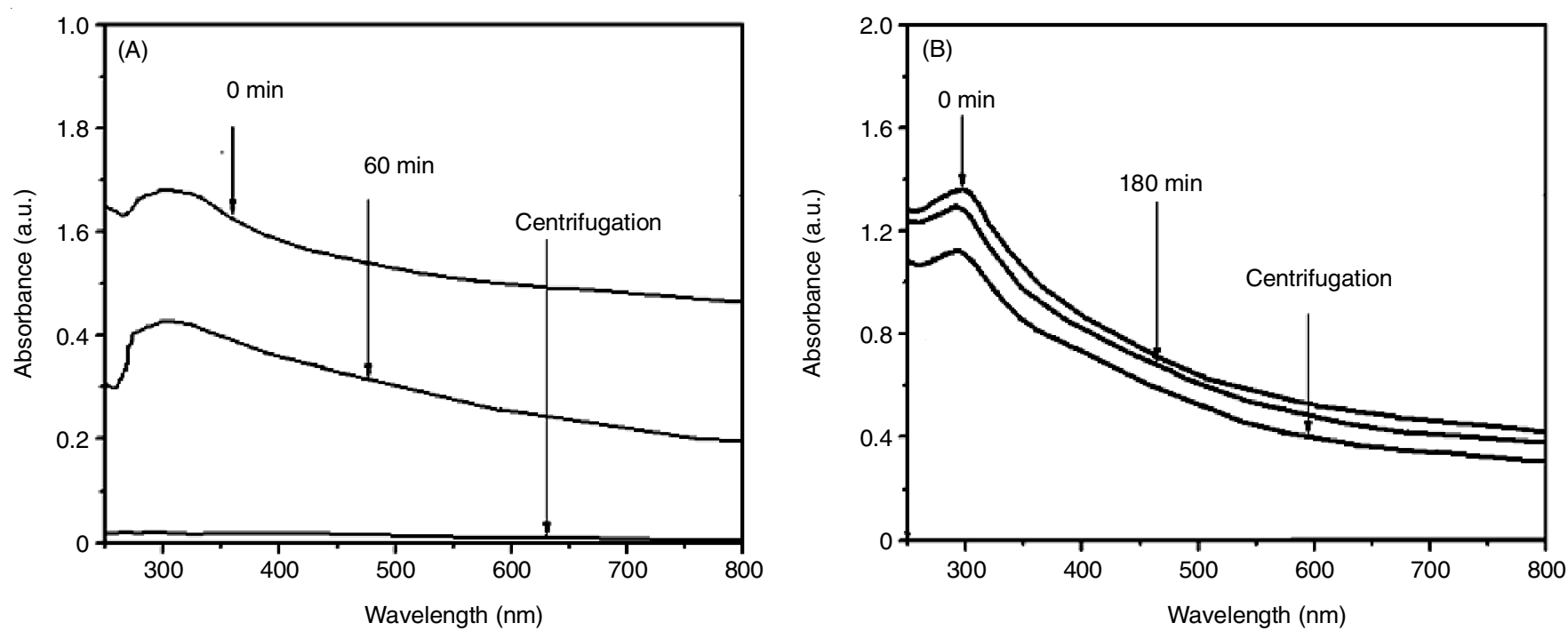

Fig. 6. UV-visible spectra of (A) HAP nanocrystals, (B) PMMA-g-HAP nanocomposites in chloroform $(0.10 \mathrm{mg} / \mathrm{mL})$

Because the HAPs accomplish to create great micro-scale concentrates in solution, thus, the bettering of the dispersal natures and the colloidal establishment of HAPs in solutions is a great challenging issue. The anchoring effect of the PMMA on the dispersion behaviour of HAPs was examined by the UV-visible absorption analysis. The HAPs and PMMA- $g$-HAP (polymerization time $9 \mathrm{~h}$ ) were independently dispersed in chloroform upon sonication for $10 \mathrm{~min}$. The UV-visible spectra of the HAPs and PMMA- $g$-HAP in chloroform are demonstrated in Fig. 6. The absorbance declined over time in the case of HAPs (Fig. 6A), which might be because of the aggregation and sedimentation of the HAP nanocrystals. When the HAPs dispersion in chloroform was underwent for centrifugation at $4000 \mathrm{rpm}$ for $5 \mathrm{~min}$, the absorbance intensity dropped to nearly zero. On the other hand, the absorbance of the PMMA- $g$-HAP decreased only a little bit even after centrifugation and the nanocomposites sustained as a finely dispersed colloidal state in chloroform (Fig. 6B). This high stability might be aroused from the steric repulsion between polymer brushes, which is administered by osmotic pressure and the affinity of the grafted polymer to the solvent.

\section{Conclusion}

Herein, we have demonstrated that the thiol lactam initiated radical polymerization protocol can be employed to polymerize MMA from the modified surface of hydroxyapatite nanocrystals with well-controlled manner. The thermal property of the PMMA upon inclusion of HAPs in the polymer matrix was found to be enhanced as suggested by TGA and DSC analyses. The XRD results indicate that the grafting of PMMA did not cause any change in crystallinity of HAP nanostructure. The GPC trace of detached polymers shows the controlled nature of the TLIRP. The synthesized nanocomposites showed excellent colloidal stability in organic solvent as observed from the UV-visible spectra and time dependent images of digital photographs. This study suggests that the precisely synthesized nanocomposites as prepared by controlled manner using TLIRP strategy could be used in biomedical, tissue engineering and regenerative medicine.

\section{ACKNOWLEDGEMENTS}

This work was supported by Nguyen Tat Thanh University, Vietnam and Pukyong National University, Republic of Korea.

\section{CONFLICT OF INTEREST}

The authors declare that there is no conflict of interests regarding the publication of this article.

\section{REFERENCES}

1. B.Q. Bao, N.H. Le, D.H.T. Nguyen, T.V. Tran, L.P.T. Pham, L.G. Bach, H.M. Ho, T.H. Nguyen and D.H. Nguyen, Mater. Sci. Eng. C, 91, 912 (2018);

https://doi.org/10.1016/j.msec.2018.07.008.

2. S.H. Mir, L.A. Nagahara, T. Thundat, P. Mokarian-Tabari, H. Furukawa and A. Khosla, J. Electrochem. Soc., 165, B3137 (2018); https://doi.org/10.1149/2.0191808jes.

3. L.G. Bach, B.T.P. Quynh, M.R. Islam and K.T. Lim, J. Nanosci. Nanotechnol., 16, 12856 (2016); https://doi.org/10.1166/jnn.2016.13651.

4. L.G. Bach, Q.T.P. Bui, X.T. Cao, V.T.T. Ho and K.T. Lim, Polym. Bull., 73, 2627 (2016);

https://doi.org/10.1007/s00289-016-1712-5.

5. L.G. Bach, B.T.P. Quynh, N.T. Thuong and V.T.T. Ho, Mol. Cryst. Liq. Cryst., 644, 175 (2017); https://doi.org/10.1080/15421406.2016.1277476.

6. M. Yazdimamaghani, D. Vashaee, S. Assefa, K. Walker, S. Madihally, G.A. Köhler and L. Tayebi, J. Biomed. Nanotechnol., 10, 911 (2014); https://doi.org/10.1166/jbn.2014.1783.

7. M. Zhang, C. Liu, J. Sun and X. Zhang, Ceram. Int., 37, 2025 (2011); https://doi.org/10.1016/j.ceramint.2011.01.045.

8. A. Szczeœ, L. Holysz and E. Chibowski, Adv. Colloid Interface Sci., 249, 321 (2017);

https://doi.org/10.1016/j.cis.2017.04.007.

9. M. Šupová, Ceram. Int., 41, 9203 (2015); https://doi.org/10.1016/j.ceramint.2015.03.316.

10. K. Szurkowska and J. Kolmas, Progr. Nat. Sci. Mater. Int., 27, 401 (2017); https://doi.org/10.1016/j.pnsc.2017.08.009.

11. M. Maas, U. Hess and K. Rezwan, Curr. Opin. Colloid Interface Sci., 19, 585 (2014); https://doi.org/10.1016/j.cocis.2014.09.002.

12. P. Terzioglu, H. Ögüt and A. Kalemtas, Mater. Sci. Eng. C, 91, 899 (2018); https://doi.org/10.1016/j.msec.2018.06.010.

13. H. Zhou and J. Lee, Acta Biomater., 7, 2769 (2011); https://doi.org/10.1016/j.actbio.2011.03.019. 
14. L.G. Bach, M.R. Islam and K.T. Lim, Mater. Lett., 93, 64 (2013); https://doi.org/10.1016/j.matlet.2012.11.051.

15. R. Barbey, L. Lavanant, D. Paripovic, N. Schuwer, C. Sugnaux, S. Tugulu and H.A. Klok, Chem. Rev., 109, 5437 (2009); https://doi.org/10.1021/cr900045a.

16. R. Matsuno, K. Yamamoto, H. Otsuka and A. Takahara, Chem. Mater, 15, 3 (2003); https://doi.org/10.1021/cm020766t.

17. J. Wei, P. He, A. Liu, X. Chen, X. Wang and X. Jing, Macromol. Biosci., 9, 1237 (2009); https://doi.org/10.1002/mabi.200900256.

18. L.G. Bach, M. Rafiqul Islam, T.-S. Vo, S.-K. Kim and K.T. Lim, J. Colloid Interface Sci., 394, 132 (2013); https://doi.org/10.1016/j.jcis.2012.11.068.

19. M.R. Islam, L.G. Bach and K.T. Lim, Appl. Surf. Sci., 276, 298 (2013); https://doi.org/10.1016/j.apsusc.2013.03.086.

20. L.G. Bach, X.T. Cao, M.R. Islam, H.G. Kim and K.T. Lim, J. Nanosci. Nanotechnol., 15, 5897 (2015); https://doi.org/10.1166/jnn.2015.10438.

21. F. Zhou, W. Liu, M. Chen and D.C. Sun, Chem. Commun., 23, 2446 (2001); https://doi.org/10.1039/b107831n

22. K. Yoshinaga, K. Fujiwara, E. Mouri, M. Ishii and H. Nakamura, Langmuir, 21, 4471 (2005); https://doi.org/10.1021/la0467786.

23. H. Cheng, G. Zhao and D. Yan, J. Polym. Sci. A Polym. Chem., 30, 2181 (1992); https://doi.org/10.1002/pola.1992.080301012.

24. X. Jiang, D. Yan, X. Zhu, J. Lin and P. Xia, J. Appl. Polym. Sci., 74, 1417 (1999); https://doi.org/10.1002/(SICI)1097-4628(19991107)74:6<1417::AIDAPP14>3.0.CO:2-5.

25. Y.H. Hu, C.Y. Chen, C.C. Wang, Y.H. Huang and S.P. Wang, J. Polym. Sci. A Polym. Chem., 42, 4976 (2004); https://doi.org/10.1002/pola.20333.
26. Y.H. Hu and C.Y. Chen, J. Polym. Sci. A Polym. Chem., 40, 3692 (2002); https://doi.org/10.1002/pola.10456.

27. L.G. Bach, X.T. Cao, V.T.T. Ho, M.R. Islam and K.T. Lim, Mol. Cryst. Liq. Cryst., 618, 120 (2015); https://doi.org/10.1080/15421406.2015.1076315.

28. M.R. Islam, L.G. Bach, J.H. Jeong, H.G. Kim and K.T. Lim, J. Nanosci. Nanotechnol., 13, 3546 (2013) https://doi.org/10.1166/jnn.2013.7278.

29. L.G. Bach, M.R. Islam, Y.H. Kim, S.D. Seo, C. Park, H.G. Kim and K.T. Lim, J. Nanosci. Nanotechnol., 13, 694 (2013); https://doi.org/10.1166/inn.2013.6929.

30. L.G. Bach, M.R. Islam, J.T. Kim, S.Y. Seo and K.T. Lim, Appl. Surf. Sci., 258, 2959 (2012); https://doi.org/10.1016/j.apsusc.2011.11.016.

31. A.M.P. Dupraz, J.R. Wijn, S.A.T. Meer and K. Groot, J. Biomed. Mater. Res., 30, 231 (1996); https://doi.org/10.1002/(SICI) 1097-4636(199602)30:2<231::AIDJBM13>3.0.CO:2-P

32. J. Chen, W. Chen, C. Deng, F. Meng and Z. Zhong, J. Polym. Sci. A Polym. Chem., 49, 4379 (2011); https://doi.org/10.1002/pola.24878.

33. Q. Liu, J.R. de Wijn and C.A. van Blitterswijk, J. Biomed. Mater. Res., 40, 358 (1998); https://doi.org/10.1002/(SICI) 1097-4636(19980605)40:3<358::AIDJBM3>3.0.CO;2-E.

34. Y. Wang, X. Zhang, J. Yan, Y. Xiao and M. Lang, Appl. Surf. Sci., 257, 6233 (2011); https://doi.org/10.1016/j.apsusc.2011.02.045.

35. W.P. Wang, H.M. Cao, G.J. Zhu and P. Wang, J. Polym. Sci. A Polym. Chem., 48, 1782 (2010); https://doi.org/10.1002/pola.23946.

36. H.J. Lee, H.W. Choi, K.J. Kim and S.C. Lee, Chem. Mater, 18, 5111 (2006); https://doi.org/10.1021/cm061139x. 